\title{
NETWORK MOBILITY SUPPORTED PROXY MOBILE IPV6
}

\author{
${ }^{1}$ Ananthi Jebaseeli SamuelRaj, ${ }^{2}$ Sundararajan Jayapal and ${ }^{3}$ Vijayaraghavan Varadharajan \\ ${ }^{1}$ Dept of Computer Science and Engineering, Anna University Coimbatore, Coimbatore, India \\ ${ }^{2}$ Principal, Pavai College of Technology, Namakkal, India \\ ${ }^{3}$ Senior Research Scientist, Infosys Labs, Bangalore, India
}

Received 2012-10-12; Revised 2013-11-28; Accepted 2014-04-26

\begin{abstract}
Proxy Mobile IPV6 (PMIPV6) is a network-based mobility management protocol, designed to keep track of individual mobile node's movement. So a mobile node can easily roam in PMIPV6 network without changing it's IP address. Network Mobility-Basic Support Protocol (NEMO-BSP), based on MIPV6, is the protocol designed for mobility management of NEMO in MPIV6 network. But NEMO-BSP cannot be directly used in PMIPV6 due to differences in the underlying protocols. To make PMIPV6 as a complete mobility management protocol, functionality of PMIPV6 should be enhanced to support network mobility in PMIPV6. This work enhances functionality of PMIPV6 and NEMO-BSP protocols and proposes a new architecture called NEMO supported PMIPV6 that supports movement of mobile nodes as well as network mobility in PMIPV6 network.
\end{abstract}

Keywords: PMIPV6, NEMO, Mobile Router, Mobile Network

\section{INTRODUCTION}

PMIPV6 (Gundavelli et al., 2008; Gundavelli, 2012) is designed to track mobility of individual mobile node. The network elements such as Local Mobility Anchor (LMA) and Mobile Access Gateway (MAG) track mobile node's movement in PMIPV6 network. So mobile nodes can be free from their own mobility management and easily roam in PMIPV6 network. But in situations where group of nodes move together like nodes in aircraft or bus, it is overhead and unnecessary to track individual node's mobility and enough to track network mobility as a whole. NEMO-BSP supports mobile network movement in MIPV6 network. But NEMO-BSP cannot be directly used in PMIPV6. Complete support of NEMO in PMIPV6 requires modification in both NEMO-BSP and PMIPV6, as per the research in this area (Bernardos et al., 2012; Jeon and Kim, 2011a).

This research work enhances PMIPV6 and NEMO-BSP protocols and proposes a new architecture called NEMO supported PMIPV6 which supports movement of individual mobile nodes as well as NEMO in PMIPV6 network.

\subsection{Issues Arise while Mobile Network Roams in PMIPV6 Network}

The issues encountered while mobile network roams inside PMIPV6 network are given below.

\subsubsection{Mobile Node's Address Changes}

In NEMO-BSP (Devarapalli et al., 2012), nodes in mobile network configure the address based on Mobile Network Prefix (MNP) advertised by MR. In PMIPV6, nodes configure their address based on Home Network Prefix (HNP) given by LMA. So movement between MIPV6 network and PMIPV6 network brings change in mobile node's address, though mobile network is inside PMIPV6 network. It contradicts the important assumption of PMIPV6 protocol, where mobile nodes in PMIPV6 network use the same address as long as the node is inside PMIPV6 network. This limits the mobile node movement between mobile network and PMIPV6 network.

\subsubsection{Load on LMA and MAG}

Let's consider a scenario where mobile network roams inside PMIPV6 network. Nodes in mobile Coimbatore, India 
network continue to stay inside mobile network and do not move between mobile network and PMIPV6 network. Mobile nodes in mobile network are really immobile in this scenario. So it is unnecessary to expose and register all nodes in mobile network with LMA. Conventional NEMO-BSP, where only Mobile Router (MR) is registered with foreign network, is enough to handle movement of mobile network. This situation urges to find the nodes which are really mobile and registers only those nodes with LMA. This will reduce the number of nodes to manage in LMA and MAG.

This research work addresses all above said problems by enhancing NEMO-BSP and PMIPV6 protocols and proposes a new architecture called NEMO supported PMIPV6 to handle movement of mobile nodes as well as NEMO in PMIPV6 network.

\section{EXISTING ARCHITECTURES}

In literature only very few works to support NEMO in PMIPV6 are found. Teraoka and Arita (2011) define a new architecture called PNEMO to support NEMO in PMIPV6. In PNEMO, Locally Fixed Nodes (LFN) are not registered with LMA, but treats both HMN and VMN as same. i.e., all mobile nodes are registered with LMA. So this architecture increases the burden of LMA by exposing HMNs. Also PNEMO has introduced four signalling messages for Binding Update and Binding Acknowledgement. These additional messages add extra signalling during mobile network movement.

Lee et al. (2010) introduce an architecture where MR acts as a MAG. when mobile network enters into PMIPV6 network; MR registers with MAG as a normal mobile node and obtains HNP from LMA. After the registration, MR acts as a MAG for nodes in mobile network and registers them with LMA. This architecture accepts MR to act as dynamic MAG. As the communication between LMA and MAG in PMIPV6 network is protected by IPSec security channel, dynamic addition and deletion of MAG requires dynamic change in preconfigured security configuration used for IPSec channel. This may lead to security issues in communication between LMA and MAG. Also this architecture has not differentiated between LFNs, HMNs and VMNs nodes. Registration of all nodes present in mobile network increases burden of LMA and introduces over burst of signaling messages during mobile network movement.

Lee et al. (2012) have defined a architecture which introduces NEMO support in PMIPV6 network.

But they talk about only Locally Fixed Nodes (LFN) in mobile network. MR obtains HNP and MNP from
LMA. It uses HNP to configure its own address and broadcasts MNP to LFNs so that LFNs can configure their address based on MNP. These study fail to explain how to handle HMNs and VMNs.

Soto et al. (2009) notifies movement of mobile network with the help of single control message. But this architecture also does not differentiate LFNs, HMNs and VMNs nodes. All nodes in the mobile network are registered with LMA. It leads to increase in load of LMA and MAG.

Jeon and Kim (2011b) propose a cost efficient network mobility scheme on PMIPV6 network. It reduces handover signaling by assigning group ID to mobile network and tracks network mobility with group ID. But this work does not differentiate between HMN and VMN. It registers all nodes in mobile network with LMA. This may increase load on LMA and MAG.

Yan et al. (2010) also introduce Network mobility support in PMIPV6. This research work concentrates on providing efficiency while nodes in mobile network undergo inter-MAG and intra-MAG movement. This research work neither concentrates on reducing handoff time nor load on LMA/MAG.

Woo et al. (2010) address the Multi tunneling problem in PMIPv6-based Nested NEMO. But they don't deal with reducing handover signaling and load on LMA and MAG.

\section{NEMO SUPPORTED PMIPV6 ARCHITECTURE}

This study proposes necessary enhancements to PMIPV6 and NEMO-BSP protocols to support mobile network movement in PMIPV6 network. Also it puts forward a new architecture called NEMO supported PMIPV6 which makes use of enhanced PMIPV6 and NEMO-BSP to support movement of mobile nodes as well as NEMO in PMIPV6 network.

\subsection{Classification of Nodes in Mobile Network}

Functionality of MR in NEMO-BSP is extended to classify nodes in mobile network as follows (i) Locally Fixed Node (LFN) which is fixed node (ii) Home Mobile Node (HMN) which is part of mobile network during boot up of MR and is assigned HNP from MR (iii) Visiting Mobile Node (VMN) which are not part of mobile network during MR boot up and joins later in mobile network. MR maintains list of nodes for each classification.

Figure 1 shows two mobile networks which are attached to PMIPV6 network and shows mobile nodes' classification list maintained by each MR. 


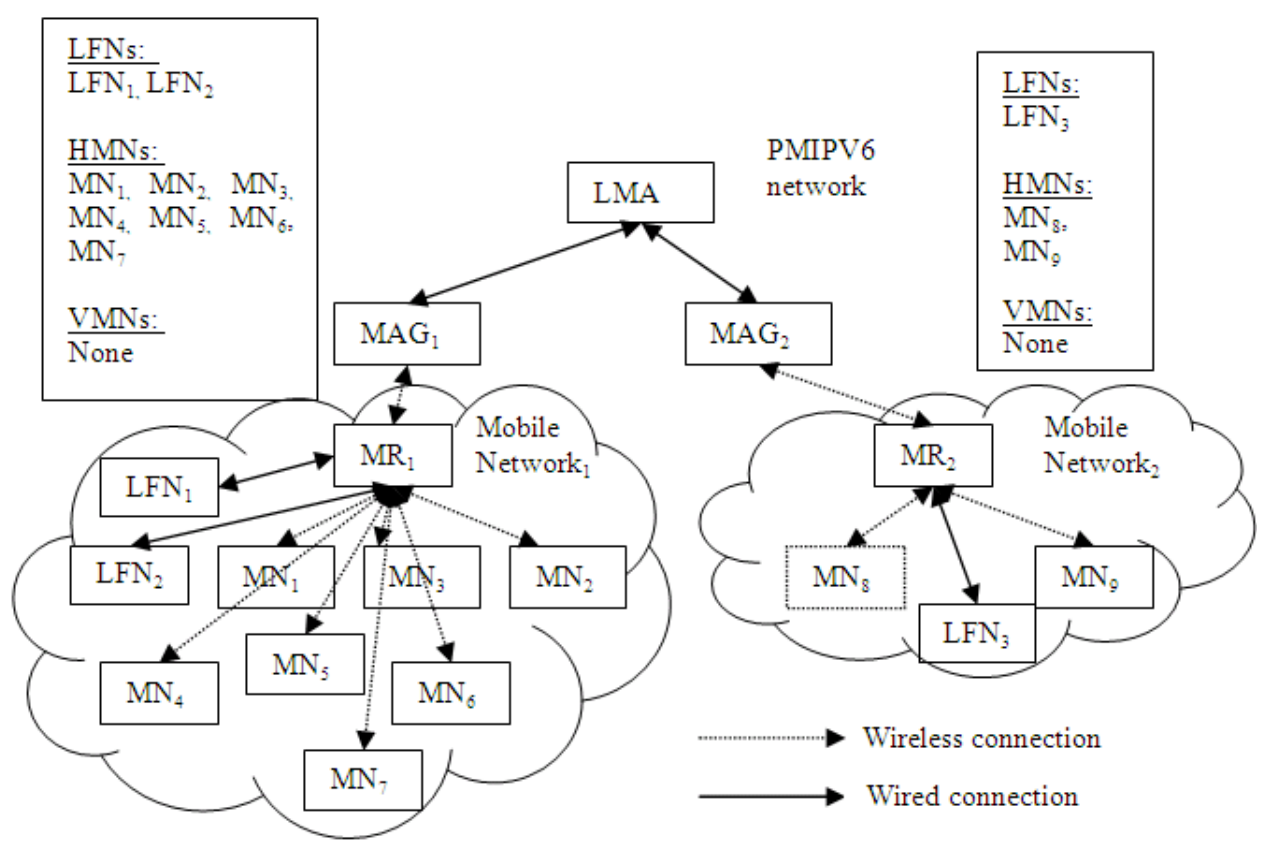

Fig. 1. Node's classification in mobile network

MR1's initial classification list is given below:

LFNs: LFN1, LFN2

HMNs: MN1, MN2, MN3, MN4, MN5, MN6, MN7

VMNs: None

MR2's initial classification list is given below:

LFNs: LFN3

HMNs: MN8, MN9

VMNs: None

As both the mobile networks are newly attached in PMIPV6 network, no VMNs are present in mobile network.

Mobile nodes start moving. MN6 and MN7 move out from Mobile network1. So MR1 deletes their entry from HMNs. MN7 joins in Mobile network2. MR2 adds MN7 in VMNs list. MN8 moves out from Mobile net-work2 and joins in Mobile network1. So its entry is de-leted from Mobile network2 and added in Mobile network1 VMNs list. After the mobile nodes' movement MR1's updated list is given below:

LFNs: LFN1, LFN2

HMNs: MN1, MN2, MN3, MN4, MN5

VMNs: MN8

MR2's updated list is given below:

LFNs: LFN3
HMNs: MN9

VMNs: None

\subsection{Registration of VMNs with LMA}

In NEMO-BSP, only MR is registered with foreign agent and obtains COA. Packets destined for nodes in mobile network are routed to MR and MR forwards the packet to the respective node. This research work extends the functionality of MR to register VMNs also with LMA and obtains HNP for VMNs. The HNP of mobile node remains same as long as the node is inside PMIPV6 network. So VMNs easily roam between mobile network and PMIPV6 network and also between two mobile networks without changing their address. As only VMNs are registered with PMIPV6 network, load on LMA and MAG is reduced.

If a mobile node moves out from mobile network and joins in PMIPV6 network for the first time, it is treated as new node and LMA assigns new address to it. Afterwards, mobile node can use the same address irrespective of its movement between mobile network and PMIPV6 network.

\subsection{Extended Functionality of MR and MAG}

In this proposed architecture, Functionality of MR and MAG is extended to act as proxy-MAG and Proxy- 
LMA respectively. Figure 2 shows NEMO supported PMIPV6 architecture. While a mobile node joins in mobile network. MR acts as proxy- MAG and sends BU with newly joined mobile node's ID to the MAG. MAG verifies authenticity of mobile nodes with the authentication server. If mobile node is authenticated to register in PMIPV6 network, it forwards BU to the LMA. LMA gives BA with the assigned HNP to MAG. MAG acts as Proxy-LMA and forwards BA to MR. MR sends Routing Advertisement (RA) with HNP to VMN. Figure 3 shows the message flow, while a mobile node joins in mobile network. When a mobile network attaches to new MAG, Same sequence is repeated for all VMNs in mobile network.

\section{PERFORMANCE EVALUATION}

The proposed architecture is implemented in NS-2. The PMIPV6 network has 1 LMA, 3 MAG, 10 mobile nodes and 1 mobile network. Simulation is conducted with different number of nodes in mobile network. Load on LMA and MAG of proposed architecture is compared with the architecture simulated with basic PMIPV6 and NEMO-BSP, which is slightly modified to register all mobile nodes in mobile network with LMA.

Load on LMA and MAG is measured with the number of nodes to be managed. Simulation is done with different number of VMNs and HMNs in mobile network.

\subsection{Simulation with Different Number of VMNs in Mobile Network}

This simulation is conducted with 5 LFNs, 10 HMNs and different number of VMNs in mobile network. When the MR boots up, only MR is registered with LMA. Gradually mobile nodes join mobile network and the number of VMNs is increased.

As the existing architecture registers all mobile nodes in the mobile network, the number of nodes to manage in LMA and MAG is same as the number of mobile nodes present in the network.

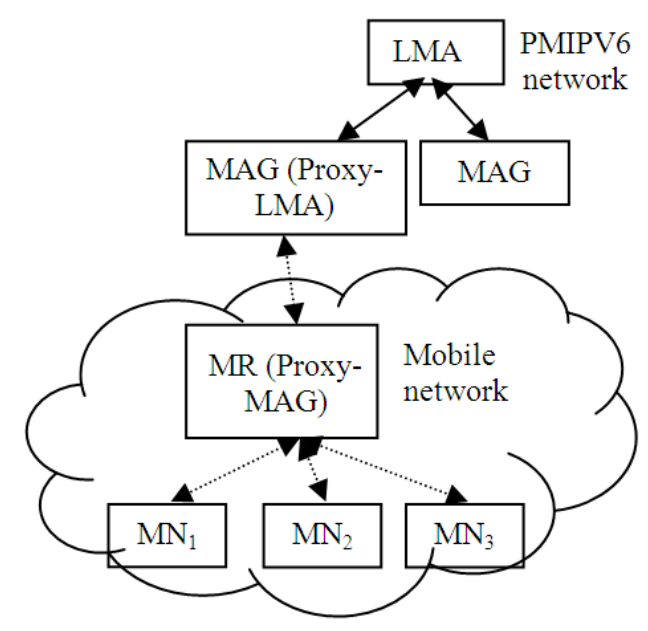

Fig. 2. NEMO Supported PMIPV6

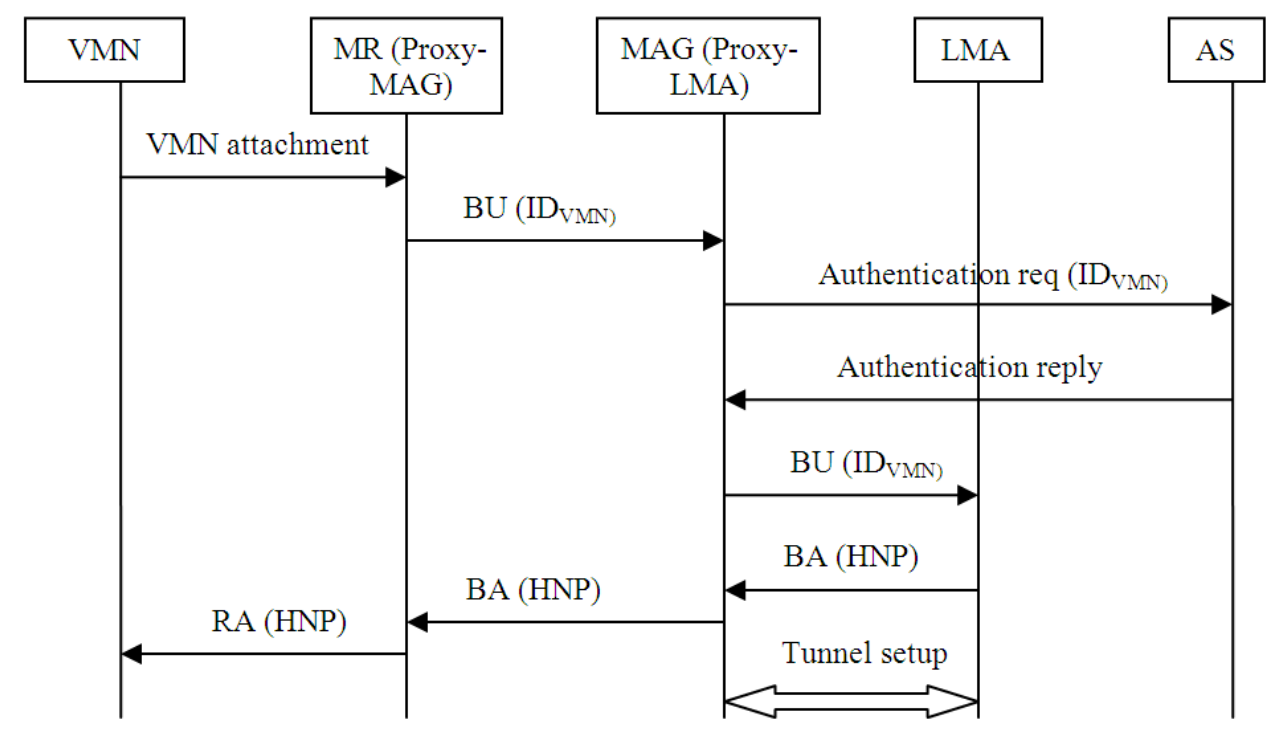

Fig. 3. Message flow during mobile node joins in mobile network 
The proposed architecture registers only VMNs which is a subset of mobile nodes present in the network. So the load on LMA and MAG in proposed architecture is always lesser than the existing architectures. Figure 4 shows the performance analysis of this scenario. Load on LMA and MAG increases as the number of VMNs increases. But the proposed architecture exhibits lesser load than the existing architecture.

\subsection{Simulation with Different Number of HMNs in Mobile Network}

This simulation is conducted with 5 LFNs, 5 VMNs and different number of HMNs in mobile network. Figure 5 shows this simulation result. The existing architecture registers all mobile nodes in the mobile network. So the load on LMA and MAG increases as the number of nodes increases. But the proposed architecture registers only MR and VMNs. As number of VMNs is kept constant in this simulation, proposed architecture shows linear graph even if the number of mobile node increases.

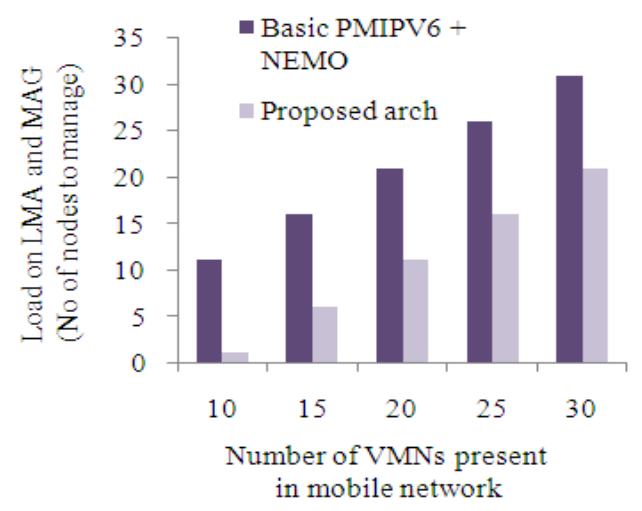

Fig. 4. Analysis of Load on LMA and MAG with different number of VMNs in the mobile network

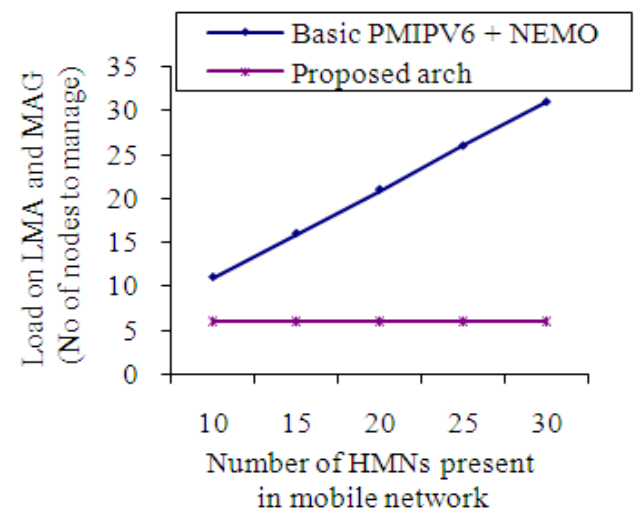

Fig. 5. Analysis of Load on LMA and MAG with different number of HMNs in the mobile network
If only LFNs and VMNs are present in mobile network, both proposed and existing architecture registers same number of VMNs with LMA. Load on LMA and MAG is same for both the architectures.

By lessening number of nodes to be registered with LMA and MAG, handoff time of mobile network is greatly reduced. From the performance analysis, it is evident that the proposed architecture exhibits better performance than the existing architectures.

\section{CONCLUSION}

NEMO supported PMIPV6 architecture enhances PMIPV6 with NEMO functionality. This research paper proposes enhancement to both NEMO-BSP and PMIPV6 to enable tracking of mobile network movement along with tracking of individual mobile node movements in PMIPV6 network. The proposed architecture classifies nodes in mobile network as LFNs, HMNs and VMNs. Only VMNs are exposed and registered with LMA. So number of nodes to manage in LMA and MAG is significantly reduced in the proposed architecture. As lesser number of nodes are exposed to PMIPV6 network, handover signaling and handoff time are reduced drastically. It is evident from the performance analysis that the proposed architecture outperforms the existing architectures.

\section{REFERENCES}

Bernardos, C.J., M. Calderon and I. Soto, 2012. PMIPv6 and network mobility problem statement. Universidad Carlos III de Madrid. IETF, Hiroshima.

Devarapalli, V., R. Wakikawa, A. Petrescu and P. Thubert, 2012. Network Mobility (NEMO) basic support protocol. Internet Engineering Task Force (IETF).

Gundavelli, S., 2012. Reserved IPv6 interface identifier for proxy mobile IPv6. RFC 6543.

Gundavelli, S., K. Leung, V. Devarapalli, K. Chowdhury and B. Patil, 2008. Proxy mobile IPv6. RFC 5213.

Jeon, S. and Y. Kim, 2011a. Cost-efficient network mobility scheme over proxy mobile IPv6 network. Instit. IET Commun. Eng. Technol., 5: 2656-2661. DOI: $10.1049 /$ iet-com.2011.0109

Jeon, S. and Y. Kim, 2011b. NEMO problem statement in PMIPv6. draft-sijeon-netext-nemo-ps-pmip601.txt.

Lee, H.B., Y.H. Han and S.G. Min, 2010. Network mobility support scheme on PMIPv6 networks. Int. J. Comput. Net. Commun., 2: 206-206. 
Lee, J.H., T. Ernst and N. Chilamkurti, 2012. Performance analysis of PMIPv6-based network mobility for intelligent transportation systems. IEEE Trans. Vehicular Technol., 61: 74-85. DOI: 10.1109/TVT.2011.2157949

Soto, I., C.J. Bernardos, M. Calderon, A. Banchs and A. Azcorra, 2009. Nemo-enabled localized mobility support for internet access in automotive scenarios. IEEE Commun. Mag., 2: 152-159. DOI: 10.1109/MCOM.2009.4939291

Teraoka, F. and T. Arita, 2011. PNEMO: A networkbased localized mobility management protocol for mobile networks. Proceedings of the ICFUN 3rd International Conference on Ubiquitous and Future Networks, Jun. 15-17, IEEE Xplore Press, Dalian, pp: 168-173. DOI: 10.1109/ICUFN.2011.5949156
Woo, M.S., H.B. Lee, Y.H. Han and S.G. Min, 2010. A tunnel compress scheme for multi-tunneling in PMIPv6-based nested NEMO. Proceedings of the WiCOM 6th International Conference on Wireless Communications Networking and Mobile Computing, Sept. 23-25, IEEE Xplore Press, Chengdu, pp: 1-4. DOI: 10.1109/WICOM.2010.5601469

Yan, Z., H. Zhou and H. Zhang, 2010. Network mobility support in PMIPv6 Network. Proceedings of the 6th International Wireless Communications and Mobile Computing Conference, Jun. 28-2, Caen, France, pp: 890-894. 